CRÍTICA, Revista Hispanoamericana de Filosofía

Vol. XX, No. 60 (diciembre 1988): 31-54

\title{
EL PRINCIPIO DE UNIVERSALIZACIÓN Y LA RAZÓN PRÁCTICA (1a. Parte)
}

Osvaldo Guariglia

Centro de Investigaciones Filosóficas CONICET y Universidad de Buenos Aires

Una de las cuestiones sin duda más debatidas en la bibliografía ética contemporánea es la que se refiere al principio de universalización y a sus aplicaciones a casos específicos, las cuales envuelven indiscutiblemente algún criterio de universalizabilidad. En la discusión de los problemas engendrados en torno a este principio en la filosofía contemporánea, se ha solido entremezclar, cuando no confundir, el principio mismo con los criterios de universalizabilidad que cada autor propone para satisfacerlo. Frente a esta tendencia, proponemos separar con claridad el principio de universalización de los criterios señalados, considerando que aquél provee un esquema lógico sobre el cual se apoyan, como sobre una garantía última, los juicios morales particulares. Con este procedimiento pretendemos estudiar la estructura del principio y los elementos que entran en su formulación; sólo luego de este examen se podrá tener una idea más precisa de lo que se requiere de un criterio de universalizabilidad para poder ser aplicado sin dar lugar a fuertes contraejemplos.

En el presente trabajo expondré, en primer lugar, (I) un esquema del principio de universalización, a fin de esclarecer no solamente su estructura lógica sino también 
los distintos conceptos que en cada caso deben ser determinados para que el principio pueda aplicarse significativamente. El análisis de cada uno de los elementos que entran en el dicurso moral: individuos humanos, propiedades de distinta índole, acciones, obligaciones y prohibiciones, pondrá en evidencia hasta qué punto la aplicación significativa del esquema de universalización presupone todo un espeso tejido de reglas semánticas, pragmáticas y lógico-prácticas previas, que constituyen el primer nivel sobre el cual se eleva, después, el juicio moral. Una vez completado este análisis, examinaré (II) la función que cumple el principio en el campo de los juicios morales y su lugar central como regla última de la razón práctica a la que confiere su peculiar forma de objetividad.

Pues uno de los conceptos que ha entrado en profunda crisis en la filosofía contemporánea ha sido, sin duda, el de "razón práctica". En efecto, mientras que la lógica y la epistemología continuaban aportando un cierto modelo, de hecho cada vez más cuestionado pero aún persistente, de "razón teórica", aquello que había sido considerado el campo tradicional de la razón práctica: la acción humana, la ética y la política, quedaba aprisionados en el dilema de reacomodarse como objeto de estudio teórico o ser abandonado para siempre al reino de lo impredecible, lo arbitrario, en suma, lo irracional. En resumen, al evaporarse, junto con los últimos restos de la filosofía de la conciencia, la obra arquitectónica de las tres Críticas, el desafío que Hume había lanzado al negar toda intervención de la razón en la moral fue renovado por las más diversas corrientes. La reconstrucción de una razón práctica, emprendida también desde diversas posiciones y con propuestas no siempre compatibles, recoge, sin embargo, con el mismo celo la herencia kantiana y su intento de elevarla por lo menos al mismo rango de validez que 
la razón en su uso teórico. El siguiente trabajo intenta presentar un examen del aporte insustituible que el principio de universalización hace para una concepción reconstructiva de la razón práctica como regla última para los juicios morales válidos.

\section{I}

1. La primera controversia con respecto al principio de universalización atañe a lo que podemos denominar su status lógico. En efecto, en el $\S 7$ de la $K p V$, en el que Kant introduce la "ley fundamental de la razón pura práctica", él se refiere a ésta con los términos 'ley', 'principio' y 'regla'. La formulación que Kant da, sin embargo, no tiene la forma de una ley, sino de un imperativo dirigido a la segunda persona: "obra de tal modo que la máxima de tu voluntad pueda valer siempre, al mismo tiempo, como principio de una legislación universal". ${ }^{1} \mathrm{De}$ acuerdo con Beck, se puede distinguir entre el 'principio' envuelto en la acción moral, la ley que expresa su estructura universal y necesaria, y la regla, en el sentido en que ésta constituye una máxima de una voluntad particular: "una regla - como dice Kant- que determina a priori la voluntad en consideración de la forma de sus máximas". Sin embargo, Kant emplea el término 'ley' también con una acepción más fuerte, que está en directa relación con su teoría de la razón práctica. En efecto, para Kant esta ley está indisolublemente unida a la conciencia de esa misma ley, es decir, a la conciencia de su existencia a priori de toda experiencia y como una proposición sintética de nuestra razón. Se trata del famoso Faktum de la razón práctica, es decir, del hecho de revelarse ella a sí misma como la Razón con una estructura universal

$1 K p V$, pp. 140-141.

$2 K p V$, p. 141; Beck, Comm., pp. 116-119. 
y legisladora. ${ }^{3}$ Creo conveniente despejar el tratamiento del principio de universalización de todo supuesto teórico o de filosofía de la conciencia como el señalado y restringirlo exclusivamente al funcionamiento lógico de aquél en los juicios morales. Desde este punto de vista, 'principio' es aquí entendido de la misma manera que lo hace M. G. Singer, es decir, como un principio presupuesto por todo juicio moral genuino, dado que constituye una parte esencial del significado de términos como 'debe' y 'correcto', pero además, como una característica esencial del razonamiento moral, porque está presupuesto en todo intento de dar una razón de un juicio moral. ${ }^{4}$

Así considerado, sólo el regreso, mediante el análisis de nuestros juicios éticos cotidianos, al principio que todos ellos presuponen y sobre el cual se sustentan -un procedimiento que desde Kant a L. Nelson ha constituido el meollo del método crítico- 5 puede aportar la única prueba posible de su existencia. Por cierto, no se trata de una prueba en sentido estricto, sino de un tipo especial que consiste, justamente, en poner en evidencia a través de los juicios particulares el principio general supuesto por todos ellos. A fin de ilustrar lo que queremos decir, proponemos el siguiente diálogo como una muestra de los razonamientos morales habituales.

A. "El concejal $\mathrm{F}$ ha obrado inmoralmente (incorrectamente) con respecto a G."

B. "¿Por qué lo dices?"

A. "Porque ha utilizado su cargo de concejal para presionar a $\mathrm{G}$ con el fin de que le vendiese un terreno que éste tenía junto a otro de su propiedad con la amenaza de expropiárselo si no accedía."

3

$K p V$, pp. 141-142.

$G E$, p. 34.

5 Cp. Nelson, EC, pp. 219-233. 
B. "¿Qué hay de incorrecto en eso?"

A. "Es incorrecto porque ni $\mathrm{F}$ ni cualquier otro en su lugar debe forzar a $\mathrm{G}$ a vender contra su voluntad."

$B$. "Pero todavía no me has dicho por qué $F$ no debe forzar a $\mathrm{G}$ a vender."

A. "Sí lo hice. Pues si se admite que $\mathrm{F}$ tiene derecho a forzar a cualquier otra persona a actuar según sus designios, entonces es necesario admitir que todos tienen el mismo derecho de hacer lo mismo que $\mathrm{F}$. Al contrario, si juzgamos que lo moralmente correcto es que esté prohibido para todos y cada uno forzar a otro a realizar una acción en contra de su voluntad, entonces esto también es válido para $F$. En otras palabras, lo que es correcto o incorrecto para una persona debe ser correcto o incorrecto para cualquier persona similar en similares circunstancias."*

Como señaló Toulmin en su ya clásica obra sobre la razón de la ética, los razonamientos morales tienen un paralelo con los razonamientos en otros campos de la argumentación justamente porque retroceden desde una

* Si bien el ejemplo ha sido imaginado por mí, sigue muy de cerca los razonamientos morales habituales utilizados tanto en los conflictos entre person as como entre sectores de la socied ad. A los ejemplos citados por Singer en $G E$, se puede agregar uno muy reciente: "La resolución 385 de la Secretaría de Energía autoriza a cooperativas, agrupaciones vecinales, empresas y organismos provinciales y municipales a construir, operar y explotar redes domiciliarias de distribución de gas. Dirigentes gremiales de Gas del Estado pidieron la derogación de esa resolución, y hasta ahí nada puede objetarse. A diario, podría decirse, se sancionan leyes, decretos o resoluciones que para numerosos sectores de la población deberían no haberse sancionado. Pero si cada uno de esos sectores decidiera emplear, dentro de sus posibilidades, medidas de fuerza o extorsivas para obtener su derogación, todo el ordenamiento institucional quedaría destruido. (...) Podría decirse, de tal forma, que entre los sucesos de Semana Santa de 1987, cuando un jefe del Ejército, al frente de tropas, intentó imponer una solución determinada en un problema de la institución militar, y la amenaza de estos dirigentes sindicales que intimaron al Gobierno a derogar una resolución einpleando como argumento la paralización de un servicio imprescindible no habría diferencia en lo esencial. La Constitución rige para todos y todos deben acatarla..." ( $L a$ Nación, editorial del 23/7/88). 
afirmación inicial a razones cada vez más generales en soporte de la primera afirmación hasta llegar a un principio de carácter universal, más allá del cual es imposible proseguir. ${ }^{6}$

Ahora bien, en el presente ejemplo este principio, que se hace explícito al final, está ya implícito en la regla general que se da como justificación para valorar la acción como incorrecta: "ni F ni cualquier otro en su lugar debe forzar a $\mathrm{G}$ a vender contra su voluntad". En efecto, como señaló acertadamente Singer, el argumento implica una generalización que va de 'no sería correcto para cualquier persona' a 'no sería correcto para nadie'. El principio de universalización explicitado al final provee la garantía última de que tal generalización es efectivamente válida. ${ }^{7}$

2. Dada la característica de este principio, es de por sí evidente que él habrá de tener necesariamente una formulación vaga y esquemática, a través de la cual, sea mediante la utilización de términos de extensión indefinida, como 'personas similares en similares circunstancias', $o$ sea mediante el uso de variables, solamente se proporcionará una regla universal que permita inferir con las apropiadas sustituciones las normas específicas aplicables a acciones particulares. En lo que sigue voy a proponer un enunciado del principio de universalización mediante una formulación esquemática que permitirá, luego, tener presentes las precisiones que se requieren para su aplicación a casos particulares.

[U] "Si F, dotado de $b$ o $c$, no debe fr o no-fr $\mathrm{G}$ o $\mathrm{H}$, dotados de $b$ o $c$ o $d$ en las circunstancias $x$ o $y$, e n t o n c e s G, dotado de $b$ o $c$, no debe fro no-fr F o $\mathrm{H}$, dotados de $b \circ c \circ d$ en las circunstancias $x \circ y$." ' $\mathrm{F}, \mathrm{G}, \mathrm{H}$ ' son variables para nombres propios de per-

- $R E$, pp. 144-145.

7 Singer $G E$, pp. 5, 15, 34,66 y passim. 
sonas. ' $b, c, d$ ' son variables para propiedades generales que delimitan clases o conjuntos de individuos humanos. Dado que se trata de propiedades relevantes con respecto a la acción, es conveniente establecer una división categorial entre ellas de acuerdo con los siguientes tipos:

(i) características permanentes y semipermanentes, por ejemplo raciales, anatómico-fisiológicas, de comportamiento, religiosas, sociales y jurídicas; (ii) capacidades, habilidades y disposiciones, por ejemplo, profesiones, entrenamientos, técnicas, etc.; (iii) roles sociales, por ejemplo, padre, madre, vendedor, comprador, administrador, empresario, obrero, etc.; (iv) roles políticos, por ejemplo, representante, diputado, secretario de estado, presidente, etc.; ( $v$ ) roles jurídicos, por ejemplo, juez, defensor, fiscal, policía, etc.; (vi) roles comunitarios, por ejemplo, delegado gremial, delegado vecinal, comisión directiva de asociaciones civiles, etc. 'No debe' es la expresión de una modalidad lógica de la acción que depende gramaticalmente del verbo modal: la expresión completa equivale a una prohibición de la acción específica en cuestión. Desde el punto de vista lógico es indiferente que se enuncie la modalidad deóntica como una obligación o como una prohibición, ${ }^{8}$ ya que éstas son interdefinibles entre sí: 'no debe no' equivale a 'debe'. Sin embargo, es necesario advertir que en el lenguaje común la expresión 'no debe' es, a veces, ambigua, ya que puede interpretarse como una prohibición o como una licencia: por ejemplo, "no debes comprar" puede interpretarse como "tienes prohibido comprar' o como 'no necesitas comprar (puedes solamente mirar la mercadería)'. En el caso de las prohibiciones, el 'no' es proléptico, ya que, en realidad, afecta a la acción, y no a la obligación: 'no debes mentir' significa 'debes no-mentir'. En el caso de las licencias, 
en cambio, la negación afecta al verbo modal: 'no debes venir, comprar, etc.' significa 'no tienes la obligación de venir, de comprar, etc.' A fin de distinguir la prohibición de la licencia, escribiré esta última uniendo la negación y el verbo modal: 'no-debe'. Por último, las razones por las que formulo el principio de universalización como una prohibición serán dadas más abajo.

' $f r$ ' es una variable para acciones que, en alguna de sus descripciones verdaderas, sean (i) transitivas con respecto a otros individuos distintos del agente, o (ii) involucren a otros individuos de modo actual, virtual o fácilmente previsible. Ejemplo de (i): 'violentar', 'forzar', 'golpear', 'descuidar', etc.; ejemplo de (ii): 'conducir un automóvil, un ómnibus, un tren, etc.' , 'evadir impuestos', 'introducir en el mercado medicamentos no experimentados', etc. A diferencia de las propiedades, que pueden tener un grado bastante grande de generalidad, las acciones requieren ser especificadas en el mayor grado posible a fin de poder establecer con precisión su aspecto moralmente relevante.

'No-fr' es una variable para omisiones que afecten directa o previsiblemente a otros individuos distintos del agente: por ejemplo, 'no socorrer', 'dejar ahogar, caer, morir, etc.', 'no apagar un fuego', 'dejar un pozo abierto en el camino sin señalar', etc. Desde el punto de vista lógico, la afirmación 'no debe $f r$ ' equivale a 'debe no-fr': en nuestro ejemplo " $F$ no debe forzar a $G$ a vender" equivale a "F debe dejar que $G$ no venda".

' $x, y$ ', por último, son variables para circunstancias, distintas de las propiedades $b, c$, etc. antes señaladas, que afectan incidentalmente la acción. Así como las propiedades $b, c$, etc. introducen cualificaciones importantes del agente y/o del paciente de la acción, las circunstancias introducen cualificaciones importantes de carácter adverbial: modifican la acción misma: 'dar un vaso lleno 
de licor a $G$ ' tiene muy distintas consecuencias si $G$ ya está ebrio, o si está a punto de conducir su automóvil, etc.

3. El análisis detallado de los términos con los que es necesario sustituir las variables que entran en el enunciado del principio de universalización, ha demostrado de qué modo la aplicación del principio a una norma específica depende de un conjunto de reglas adicionales, de carácter semántico y pragmático, que el principio simplemente presupone. La discusión de algunos de los problemas de interpretación que afectan a cada uno de los términos generales examinados habrá de mostrar hasta qué punto será necesario apelar a criterios ajenos al principio mismo para poder satisfacer adecuadamente los requisitos de su funcionamiento.

En primer lugar es necesario señalar que la restricción de las variables F, G, H a 'personas' está lejos de ser evidente. En efecto, el intento de conectar el principio de universalización con el concepto de 'persona' se remonta al mismo Kant, a pesar de lo cual hasta el momento ha sido imposible aportar una conexión lógicamente satisfactoria entre ambos. En términos generales, se puede considerar a los filósofos divididos en dos grandes campos: los que consideran que el principio es autosuficiente para determinar quién es una persona, y los que sostienen que no lo es y que, por lo tanto, se hace necesario recurrir a un criterio adicional. En efecto, desde cierto ángulo el principio provee una definición implícita de 'persona', de la siguiente manera: "Si F, G, H, son 'personas', entonces [U]", pero esta forma de interpretar el principio introduce una importante restricción que no todos sus defensores aceptarían. Para Nelson, por ejemplo, el principio iba más allá de las personas e involucraba a 'todos los portadores de intereses', una clase que incluía también a los 
animales. ${ }^{9}$ En realidad, en este punto es donde se separan las interpretaciones de los dos grupos antes mencionados: para el primero de ellos, compuesto entre otros por Kant, Nelson y Hare, el principio de universalización posee un criterio intrínseco para establecer con claridad los 'sujetos morales' a los cuales se aplica $;{ }^{10}$ para el segundo, en cambio, el principio expresa un requisito esencial del tratamiento de las personas como personas pero no aporta por sí mismo un criterio para distinguir qué individuos deben ser considerados personas y quiénes no. ${ }^{11}$

Probablemente haya detrás de esta división dos modos enfrentados de concebir el funcionamiento semántico de términos como nombres propios o de clases naturales o cuasi-naturales, como 'personas'. Para los primeros, cualquier aplicación del principio determinará, mediante una combinación de propiedades y relaciones universales similar a una descripción definida, la clase de individuos para los que tiene validez. Para los segundos, en cambio, este procedimiento podrá aportar, a lo sumo, una definición tautológica, como la ya indicada más arriba, pero no nos dará ningún criterio para fijar pragmáticamente la referencia de $F, G$ y $H$. En otros términos, el criterio para distinguir a qué individuos es posible considerar personas y a quiénes no, está provisto por un uso social e institucional del lenguaje que remite, inevitablemente, a las circunstancias históricas de determinadas comunidades morales y linguísticas.

A mi juicio, existe un argumento adicional en favor de esta última posición, que es el siguiente. Si bien el principio de universalización no provee un criterio para decidir quiénes son personas, provee, en cambio, un cri-

\footnotetext{
9 Nelson, EC, pp. 68-69.

10 Cp. Hare, $M T$, pp. 108 ss.

11 Cp. Singer $G E$, pp. 233-37; Silverstein $T P$, pp. 64-66.
} 
terio seguro para determinar quiénes no son considerados personas en una determinada sociedad histórica. En efecto, del empleo anterior que hicimos de [U] para la definición implícita de persona, se sigue lógicamente que, si [U] no tiene lugar para alguna sustitución individual ('Juan Pérez') o combinada con alguna propiedad (JP 'de piel negra' o 'hispanoparlante'), entonces ese individuo o esa clase de individuos no son personas. En todas las épocas históricas hasta la actualidad ha habido alguna combinación de propiedades que cierta sociedad consideraba como no-persona: bárbaros, plebeyos, negros, judios, etc. Desde el punto de vista normativo, la consecuencia inmediata de esta decisión consiste, justamente, en quedar excluido de las relaciones de simetría que establece el principio de universalización. En las precisas palabras del mayor historiador contemporáneo de la esclavitud antigua, M. I. Finley, "cuando los jurisconsultos romanos definían al esclavo como alguien que estaba bajo el dominium de otra persona, se servían del término quintaesencialmente posesivo dominium. No les disuadía la cualidad humana del esclavo... Tampoco a los millones de propietarios que los compraban y los vendian, los hacian trabajar en exceso, los castigaban y torturaban, y a veces los mataban, ni más ni menos que como han venido haciendo los millones de propietarios de caballos a lo largo de la historia (...) Que un propietario de esclavos no ejerciera todos sus derechos sobre su propiedad esclava era simplemente un acto unilateral de su parte, nunca obligatorio, siempre revocable... (...) En cuanto a promesas(...) ningún amo podía ser llevado a los tribunales basándose en la promesa hecha a un esclavo". ${ }^{12}$

Situaciones como la descrita por Finley ilustran sobre una posible conexión entre el principio de universa-

Finley, EA, p. 93. 
lización y el reconocimiento de no-personas como personas, que exploraremos más tarde. Por ahora las observaciones apuntadas bastan para afirmar que los candidatos a sustituir las variables $F, G, H$ en el enunciado del principio deben ser provistos apelando necesariamente a un criterio sintético (semántico o pragmático) distinto de aquél.

4. En segundo lugar, es necesario reflexionar con cierta amplitud sobre el carácter de las propiedades involucradas en los juicios morales. Ha habido una tendencia general a menospreciar la importancia de estas propiedades, enfatizando, en cambio, la de las modalidades deónticas y la de las acciones. La posibilidad de abstraer más fácilmente las reglas lógicas implícitas en el uso de los verbos modales (operadores deónticos) y de los verbos de acción (como una especie de las relaciones) llevó a oscurecer sistemáticamente la importancia de las propiedades, mucho más difíciles de reducir a relaciones meramente sintácticas. Al contrario, a través de ellas penetra en el horizonte del juicio moral la multiplicidad y opacidad del mundo fáctico, con sus asimetrías, conexiones causales y determinaciones histórico-narrativas.

Ahora bien, al hablar de propiedades se hace inevitable intentar alguna caracterización de éstas que precise el campo de las entidades de las que se trata. Este requisito sería, sin duda, innecesario si nuestro presente cometido fuera exclusivamente lógico, ya que en este último caso el único aspecto importante se relacionaría con la mayor o menor extensión de los términos. Desde el punto de vista ético, en cambio, la extensión de una determinada propiedad pasa a segundo plano al considerarse los aspectos relevantes de una situación, mientras que lo que cobra importancia es la cualidad de las mismas. Sin embargo, tan pronto hablamos de los qualia nos enfrentamos a dos problemas ante los que es ineludible tomar posición: (i) la 
clasificación categorial de sus significados, y (ii) la naturaleza y forma de su referencia. Discutamos cada uno de ellos por separado.

(i) Clasificación categorial del significado. La primera distinción radical entre los significados de los términos, que constituye simultáneamente una clasificación de las propiedades en primarias y derivadas (o secundarias), se la debemos a Platón. ${ }^{13}$ En efecto, al establecer una estricta separación entre "los entes que se dicen siempre por sí mismos" y "los entes que se dicen siempre con relación a otros" introdujo el primer criterio no meramente lingüístico para diferenciar las propiedades por el carácter de su significado. La interpretación precisa de la distinción platónica está lejos de ser una cuestión cerrada entre filólogos e historiadores de la filosofía. Habitualmente se sostiene que Platón coloca dentro del primer género de entidades propiedades tales como 'hombre', 'árbol' o 'triángulo', mientras que bajo el segundo caerían no solamente nuestros relativos, i.e. 'mayor', 'doble', etc. sino también atributos tales como 'rojo' o 'blando', que admiten gradaciones y suponen, implícitamente, un segundo término de comparación. ${ }^{14}$ No deseo, ni por un momento, entrar en la liza en torno a lo que Platón realmente propuso; pretendo mostrar, solamente, un rasgo del platonismo que habrá de retornar hasta en nuestros mismos días. En efecto, sea cual fuera la interpretación última, algo es claro: Platón pretende introducir una drástica diferencia entre términos que poseen un significado 'completo' y que remiten a entidades perfectas y términos que poseen un significado 'incompleto', es decir, que requieren de otro para poder ser entendidos, los cua-

13 Sofista, 255c-d.

14 Cp. G. E. L. Owen, "A Proof in the Peri Ideon" (1957), en R. E. Allen, ed., Studies in Plato's Metaphysics, Londres, Routledge \& Kegan Paul, 1965, pp. 302-312. 
les remiten a entidades deficientes en comparación con las primeras.

No otra es la distinción que reintroduce Galileo cuando propugna desechar las cualidades sensoriales, sabor, color, sonido, etc. como meramente subjetivas y carentes de entidad y conservar aquellas que determinan el límite, la forma, el tamaño, el lugar, etc., es decir, sólo las que son susceptibles de medición o composición geométrica. ${ }^{15}$

La más reciente versión del platonismo semántico, por último, fue protagonizada por el Círculo de Viena, especialmente por R. Carnap, mediante la imposición del criterio de verificación a las palabras como prueba incontrovertible de su significado. De este modo, toda palabra se encontró sometida a una doble tensión: por un lado, la necesidad de equiparar su significado con determinadas sensaciones elementales, no más reductibles a otras; por el otro, la necesidad de estar incorporada a un lenguaje sintáctico de modo que su significado no fuese independiente de las proposiciones protocolares de las que formase parte, cuyas condiciones de verdad debían estar previamente estipuladas. De acuerdo con ello, aprenhender el significado de un término equivale no solamente a aprenhender la propiedad en cuestión, sino también a poseer el conocimiento de las reglas sintácticas por medio de las cuales una proposición formada por la predicación de esa propiedad podía ser derivada de otras proposiciones más el conocimiento fáctico de regularidades empíricas que asocien la presencia de esa propiedad con la presencia o ausencia de otras propiedades, más la capacidad de poder verificar mediante las adecuadas predicciones las condiciones por medio de las cuales se pueden confirmar o refutar esas regularidades, etc. ${ }^{16}$

15 Cp. E. Cassirer, Das Erkenntnisproblem in der Philosophie und Wissenschaft der neueren Zeit, Darmstadt, WB, 1974, t. I, pp. 390-93.

${ }^{16}$ Cp. Las exposiciones críticas de esta teoría semántica de H. Putnam, 
De esta manera se impuso una distinción categorial dentro de la semántica misma de los términos que estaría cargada de consecuencias para el desarrollo de las ciencias sociales y de la ética durante el transcurso del presente siglo: la distinción entre el significado descriptivo de las palabras y el 'significado' no descriptivo (valorativo, metafísico, emotivo, etc.) de ellas. ${ }^{17}$ No constituye un obstáculo para sostener que existió (y existe aún) un platonismo semántico ampliamente compartido -bautizado ahora por Putnam 'One True Theory'18 - el hecho de que diferentes filósofos dentro de esta tradición explicaran de maneras diferentes el 'significado' de los términos no descriptivos: desde su inexitencia lisa y llana en el mundo, ${ }^{19}$ hasta su aceptación como transmisores de una carga emotiva, dinámica $o$, simplemente, una recomendación. ${ }^{20} \mathrm{La}$ distinción semántica fundamental entre términos que refieren a entidades en el mundo y términos que no tienen referencia y que, por lo tanto, carecen en última instancia de objetividad, ya que no es posible establecer para ellos las apropiadas condiciones de verdad, se ha mantenido invariable. Una consecuencia inmediata de la adopción de una teoría semántica como esta consistiría en la reducción drástica de las seis clases de propiedades que hemos establecido más arriba a solamente dos: características físicas, empíricamente comprobables, por un lado, y 'propiedades' que encubrirían meras estipulaciones $o$, a lo sumo, hipótesis probabilísticas sobre covariancias de comportamientos, por el otro.

$M R L$, pp. 196-271; MMS, pp. 97 ss. y $R T H$, passim; por cierto esta teoría no es exclusiva del positivismo lógico, sino que también fue adoptada, entre otros, por el primer Wittgenstein y Popper.

17 Cp. Guariglia, ILV, cap. 1; Hudson, $M M P$, cap. 2.

${ }^{18}$ Cp. RTH, p. 73.

19 Wittgenstein, Tractatus 6.41 .

${ }^{20}$ Hare, $L M$ 7.4, 7.5; Urmson, ET, pp. 88 ss. 
La posición alternativa con relación a los significados de las propiedades involucradas en los juicios del lenguaje natural tiene, también, su punto de partida en la filosofía griega. Fue formulada por Aristóteles en el primer libro de los Tópicos justamente como una teoría semántica destinada a evitar equivocidades en las premisas dialécticas, esto es, que constituían los puntos de partida de una refutación del adversario por medios argumentativos. En la exposición de Tópicos I 9 Aristóteles presenta los términos que pueden entrar en una proposición divididos entre distintos 'modos de significación' que son excluyentes entre sí. Cada uno de estos 'modos de significación' de las palabras corresponden a una conexión de sentido que, a su vez, es puesta de manifiesto por medio de su correlación con una cierta interrogante: '¿qué?', '¿cómo?' '¿cuánto?', ‘dónde?', etc. Cada una de estas preguntas determina tácitamente un conjunto limitado de respuestas posibles: 'un hombre', 'blanco', 'un codo', 'en Atica', etc. Estas respuestas, a su vez, pueden ser el sujeto de una nueva pregunta, '¿quée?', la cual apunta a la explicitación de la definición del nombre en una más amplia combinación de significados. Las respuestas habrán de mantenerse dentro del mismo modo de significación para poder preservar una conexión de sentido: cualquier transgresión a esta regla implícita produce, en consecuencia, un sin-sentido. De este modo, Aristóteles introdujo su famosa distinción categorial justamente como una estratificación del significado de las palabras y una consiguiente estratificación de las propiedades que entran en los juicios independientemente de toda distinción ontológica entre las respectivas referencias. Su propuesta pone por primera vez al descubierto la trama de reglas semánticas de uso y aplicación signi- 
ficativa de los términos que está en juego en la práctica discursiva del lenguaje. ${ }^{21}$

Luego de un largo eclipse una nueva versión de esta actitud semántica fue reintroducida por el propio Wittgenstein por medio de una crítica tan sostenida como consistente a su propia posición en la época del Tractatus. $^{22}$

A la reducción categorial de todos los términos del lenguaje a nombres correspondientes a hechos elementales, se contrapone ahora la multiplicidad de funciones del lenguaje, multiplicidad que se manifiesta en la gran variedad de juegos de lenguaje que se ocultan tras cada término. ${ }^{23}$

Wittgenstein enfatiza de un modo especial la diferencia que separa la significación (Bedeutung) de una palabra del portador de la palabra ( Träger), lo que hoy llamamos su referencia. ${ }^{24} \mathrm{La}$ significación de la palabra está ahora constituida por su uso en el lenguaje, es decir, por las reglas profundas que llevan a los hablantes de una lengua a realizar determinadas combinaciones de términos y a evitar otras. La tarea de poner de manifiesto, caso por caso, estas reglas de uso de un término, que se ocultan tras la engañosa apariencia de uniformidad de las palabras alineadas, como en un diccionario, unas junto a otras, es lo que Wittgenstein ha denominado la gramática profunda

21 Para una exposición detallada remito a Guariglia, "El carácter original de las categorías en los Tópicos de Aristóteles", Journal History of Philosophy 19 (1981) pp. 1-20, espec. pp. 13-18.

22 La similitud entre la teoría semantica del último Wittgenstein con la de Aristóteles, especialmente en sus obras más dialécticas, Tópicos, Ética, etc. fue señalada por R. Bambrough, $A J$, pp. 170 ss., y desarrollada en detalle por J. D. G. Evans, $A C D$. Para la relación entre el Tractatus y la obra posterior, Kenny, $W$, pp. 139 ss. y Apel, TP, I pp. $335 \mathrm{~s}$.

${ }^{23}$ Cp. $P U$, pp. 23-27; $P G$, pp. 56-58, 66-67.

$24 P U$, p. $40,43$. 
por oposición a la gramática superficial que regula su uso. ${ }^{25}$

A pesar del rechazo de todo intento de sistematización de las distintas palabras y sus usos, Wittgenstein establece en la $P G$ la existencia de especies de palabras (Wortart) distintas de acuerdo con el modo de su aplicación: "Y se podría — señala- distinguir muy bien en la gramática usual entre las especies de palabras siguientes: 'palabra de forma', 'palabra de color', 'palabra de sonido', 'palabra de materia', etc." 26 Es claro que él apunta aquí hacia un aspecto del lenguaje muy similar al que Aristóteles aludía con su teoría semántica de las categorías como especies o modos de significación. Se trata, en última instancia, de las conexiones de sentido que son reguladas por las reglas de uso de los términos, las cuales permiten, a su vez, establecer agrupaciones y paralelos entre ellas que configuran esas constelaciones de significados que Aristóteles llamó 'analogías' y Wittgenstein 'parecidos de familia'. Una concepción semántica de esta naturaleza constituye lo que podríamos denominar una gramática de la inteligibilidad, justamente porque presupone tanto un significado público de las palabras como un diferente grado de inteligibilidad de éste para los diferentes usuarios del lenguaje en relación con el mayor o menor dominio de las reglas de uso que depende de circunstancias como la formación individual, el grado de especialización de la terminología, etc.

Una distinción categorial, como la propuesta más arriba (I.2), de las propiedades que entran en un juicio moral entre seis clases distintas, supone precisamente un compromiso con una actitud semántica similar a la de la gramática de la inteligibilidad desarrollada por ambos

$P U$ I, p. 664; Kenny, $W$, pp. 148-50.

26

$P G$, p. 61 . 
pensadores. En efecto, tanto las condiciones para sostener la atribución de determinadas propiedades a determinados sujetos como las consecuencias que se siguen de la atribución de esas propiedades remiten a convenciones previas que regulan tanto la aplicación de los términos como las expectativas que surgen de esa aplicación con respecto a las acciones y demás relaciones con el mundo físico y social que los poseedores de esas propiedades habrán de tener. Por cierto, la existencia de tales convenciones que regulan la gramática de la inteligibilidad de los términos no equivale a establecer la existencia de significados regulados una vez y para siempre en el interior de un determinado lenguaje. Los márgenes dentro de los que se mueve la aplicación inteligible de la palabra en cuestión son flexibles y admiten una amplia zona de fluctuación. Sin embargo, ésta tiene un límite bastante preciso, que está dado por la imposibilidad de transgredir el ámbito de aplicación compartido de un término para pasar a un uso privado del mismo. ${ }^{27}$

En este punto es en el que se inserta la otra gran cuestión que hemos dejado abierta con relación a las propiedades, a saber: el problema de la referencia.

5. (ii) Naturaleza y forma de la referencia. La semántica posterior a Wittgenstein ha presentado hasta el momento cuatro grandes líneas interpretativas de la relación entre el sentido y la referencia que recogen, a mi juicio, algunos de sus resultados: el programa intencionalista de P. Grice; el programa convencionalista de J. Searle, que se propone hacer confluir la teoría de los actos lingüísticos con la semántica; la hermenéutica trascendental de K. O. Apel, quien se propone aunar ambos intentos preceden-

27 Wittgenstein, $P U$ pp. 272, 273, 510. Para la relación entre el argumento del lenguaje privado y las teorías de la significación, cp. el instructivo análisis de E. Villanueva, "Las teorías del significado y el argumento contra el lenguaje privado", Revista Latinoamericana de Filosofía 9 (1983) pp. 195-210. 
tes desde una perspectiva trascendental y comunicativa, y, por último, la teoría causal de la referencia, inaugurada por S. Kripke y H. Putnam, que ha introducido puntos de vista sumamente originales y ha renovado una perspectiva 'realista' en semántica. ${ }^{28}$

Ahora bien, nuestro interés inmediato en la relación entre sentido y referencia de los términos que entran en un juicio moral, en especial de aquellos que expresan propiedades, está centrado en el aspecto que Apel presenta de la siguiente manera: "[The] utterer's meaning is made possible by his sharing the so called public timeless meaning of types of linguistic utterances. Now this latter aspect of meaning is itself not only dependent on linguistic conventions,(...) but also on what we have called reference to things in a broad sense." ${ }^{29} \mathrm{El}$ problema consiste, justamente, en poder incluir este aspecto de la referencia a las cosas nuevamente en el interior del significado de un término sin recaer en un reduccionismo similar al verificacionista. Apel propone para ello una nueva versión de la explicación operacional, introducida por C. Pierce, del significado, que consiste en "intentar anticipar, una y otra vez, el posible consenso en torno a los 'intérpretes lógicos últimos' de los signos por una comunidad indefinida de intérpretes" ${ }^{30}$ Esta propuesta, sin embargo, parece seguir de cerca la identificación del significado con un racimo de propiedades, las que en su conjunción delimitan la extensión del término a definir. En otras palabras, lo que los intérpretes ideales harían, sería establecer el alcance 'conceptual' de una determi-

28

Cp. Searle, $S A$, pp. 42 ss., 146 ss.; Apel, ICR, pp. 90 ss.; Kripke, $N N$, espec. pp. 106 ss.; Putnam, "The meaning of the meaning", $M R$, pp. 215 88.

29 ICR, p. 104.

$30 I C R$, p. 110; TP 2, p. 349 . 
nada palabra, teniendo en cuenta los datos apropiados y la correspondiente teoría.

A esta forma de entender la referencia de los términos, al menos de un conjunto de términos que incluye los nombres propios, las especies naturales, etc., se ha opuesto la teoría causal de la referencia. En efecto, tomando como modelo la forma de referir de los nombres propios, Kripke ha desarrollado el concepto de 'designador rígido', i.e., de un designador que se refiere al mismo individuo en todos los mundos posibles. De este modo, Kripke opone los designadores rígidos a las (posibles) descripciones definidas cuya extensión, en el mundo actual, puedan coincidir con la del nombre propio. Mientras que José de San Martín referirá siempre al mismo individuo procedente de la unión de un determinado par de gametas, las descripciones "el vencedor de Chacabuco y Maipú", válidas para el mundo actual, pueden variar en otro mundo posible. La aplicación de esta distinción a los nombres de especies naturales, 'gatos', 'tigres', o de términos de sustancias, como 'agua', 'oro', 'pirita', etc., así como también a fenómenos naturales, como 'calor', 'luz', 'rayo', etc. produce una muy interesante disociación entre la referencia de estos términos, que es invariable, y aquellas propiedades superficiales que proveen la manera en que la referencia correspondiente es fijada. De este modo se elimina la analiticidad entre la referencia del término, que es una muestra de la especie o sustancia en cuestión, y el racimo de propiedades, que puede variar según el grado de conocimiento y de pericia de los hablantes, mediante el cual se fija la forma de la referencia. ${ }^{31}$ Putnam, por su parte, ha extendido esta concepción semántica tanto al lenguaje natural como al lenguaje de las teorías científicas. Con respecto al lenguaje natural, en el que

31 Kripke, $N N$, pp. 134-35; Schwartz, $N K$, pp. 26 ss. 
normalmente se expresan los juicios morales, Putnam propone dos rasgos esenciales para la comprensión del significado de los términos: en primer lugar, la universalidad de la división del trabajo lingüístico; en segundo lugar, la indexicalidad de todos los términos referenciales del lenguaje, incluidos los términos de artefactos ('lápiz') y los teóricos ('electrón') . La tesis sostenida por Putnam mediante la división del trabajo lingüístico consiste en afirmar que el hablante medio de un lenguaje, al adquirir un término, no fija individualmente la extensión de su referencia, sino que incorpora un nuevo estereotipo que refiere indexicalmente a la muestra o paradigma (un perro, un álamo, un trozo de granito, de pirita, de mármol travestino, etc.) mediante el cual hizo el aprendizaje del término. Al contrario, fijar con exactitud la referencia de un término constituye la tarea de un grupo de expertos, quienes mediante las consiguientes pruebas empíricas y científicas, establecerán en cada caso la extensión. Por lo tanto, la extensión de los términos solamente puede ser fijada socialmente, nunca individualmente. Las operaciones llevadas a cabo por los expertos apra fijar la referencia, así como la transcripción simbólica en un lenguaje más exacto que pone de manifiesto la microestructura de un trozo de sustancia, de una célula, etc., nunca constituye una descripción analítica del término. ${ }^{32}$ Utilizando un ejemplo familiar, el vocablo 'delfín' siguió refiriéndose indexicalmente a la misma especie de seres acuáticos aún después que se descubriera que no eran peces sino mamíferos.

32 Putnam, MR, pp. 227 ss.: Un ejemplo de las consecuencias de esta concepción para los términos teóricos, es el siguiente: el término 'electrón' que $\mathbf{N}$. Bohr introdujo en su teoría de los años 1900 refiere la misma entidad que en la física actual, a pesar de que todas sus demás descripciones no coincidan exactamente con las propiedades que la teoría física de hoy (y la del mismo Bohr en época posterior) atribuye al electrón. Cp. Putnam, MMS, pp. 22 ss. 
La distinción categorial de las propiedades, presentada en I 3, a las que podemos agregar ahora también aquellas otras que, como las que señalan circunstancias $(x, y$, en nuestro esquema), tienen un tipo de indexicalidad aún más manifiesta, ya que remiten deícticamente a contextos acotados (en tal estado, a tal hora, en tal lugar, etc.), permite una clasificación similar también con respecto a las referencias. Es claro que esto es sin más evidente para las especies naturales humanas: 'negro', 'blanco', 'amarillo', i.e., que remiten a características antropológiconaturales. También con relación a términos como 'cojo', 'manco', 'jorobado', 'tullido', 'cardíaco', 'mongólico', etcétera, que se refieren a características anatomico-fisiológicas de cada individuo en especial. A mi juicio, no existe diferencia alguna en el empleo de los demás términos que refieren a capacidades, roles sociales, políticos y/o jurídicos. Aprendemos términos tales como 'médico', 'maestro', 'profesor', 'policía', 'juez', de un modo indexical, como referencia fija a un determinado profesional, etc., que aparece como modelo con ciertos rasgos vagos muy generales asociados a él, a pesar de que la determinación precisa dependa, como por ejemplo en el caso del médico y del juez, de complejas disposiciones tanto de índole técnica como jurídica. Aún roles tales como 'comerciante', 'industrial', etc. contienen este núcleo referencial básico, aún cuando las descripciones que en cada caso se hagan de ese significado básico puedan variar drásticamente de uno a otro caso, dependiendo fuertemente de tradiciones culturales, estructuras sociales, carácter general de la economía, etc.

El hablante medio de un lenguaje natural posee, por lo tanto, a través de su competencia en el uso de los términos y de su conocimiento de aquellos representantes paradigmáticos de las propiedades que tienen una dirección referencial hacia el mundo natural, social, econó- 
mico y político que lo rodea, los datos básicos que entran en un juicio moral, datos que permiten, tácitamente, inferir determinadas posibilidades y excluir otras con respecto a lo que se puede esperar de la atribución de ciertas propiedades a ciertos sujetos. Como veremos de inmediato, este mismo procedimiento también es válido para las acciones que entran en el juicio moral, ya que las propiedades señaladas y las acciones están unidas conceptualmente. Ahora bien, de nuestra presentación se sigue que el hablante medio de un lenguaje posee a través de su conocimiento del significado referencial de los términos sólo una limitada visión del universo de cosas y hechos sociales que lo rodea. El carácter de público y abierto de los sign.ificados así como la dirección hacia los hechos que constituye la indexicalidad de los mismos, le permitirá profundizar el conocimiento de las propiedades descriptivas que fijan con mayor precisión y amplitud la referencia de un término, siempre que esto último sea necesario. Es, justamente, este otro miembro de la identidad, que no solamente comprende la conjunción de las múltiples propiedades superficiales sino también el conocimiento técnico de la fórmula científica que simboliza la microestructura de un hecho natural o las reglas constitutivas (leyes, reglas de funcionamiento, etc.) de un hecho social, el que en definitiva es captado por la discusión racional de los intérpretes lógicos últimos en condiciones ideales de conocimiento de todos los datos y teorías pertinentes que propone Apel. Pero, con esta comprobación estamos abandonando el campo exclusivamente semántico para adentrarnos en las condiciones epistémicas del juicio moral, de las que hablaremos más tarde. 